\title{
Efeitos da suplementação de $\beta$-hidroxi- $\beta$-metilbutirato sobre a força e a hipertrofia
}

\author{
Effects of $\beta$-hydroxy- $\beta$-methylbutyrate \\ supplementation on strength and hypertrophy
}

Thiago da Silveira ALVARES ${ }^{1}$

Cláudia de Mello MEIRELLES ${ }^{1,2,3}$

RE S U M O

o $\beta$-hidroxi- $\beta$-metilbutirato é um metabólito da leucina estudado devido aos seus efeitos anticatabólicos e possíveis implicações sobre os ganhos de força e massa muscular associados ao treinamento contra-resistência (conhecido como musculação). O objetivo deste trabalho foi revisar a literatura referente à suplementação de $\beta$-hidroxi- $\beta$-metilbutirato e os seus efeitos sobre força e hipertrofia em adultos jovens e idosos iniciantes ou treinados em treinamento contra-resistência. Estudos em indivíduos iniciando um programa de treinamento contra-resistência sugerem que a suplementação diária de 1,5 a 3,0g de $\beta$-hidroxi- $\beta$-metilbutirato pode trazer benefício ergogênico durante as primeiras quatro ou cinco semanas. Entretanto, à medida que o programa de treinamento evolui, tais efeitos não permanecem e os ganhos de força e massa muscular são proporcionados apenas pelo treinamento contra-resistência. Em indivíduos treinados, os resultados parecem não ser os mesmos, uma vez que, desde o início da suplementação, o $\beta$-hidroxi- $\beta$-metilbutirato não demonstra qualquer benefício adicional aos induzidos pelo treinamento contra-resistência. Alguns efeitos do $\beta$-hidroxi- $\beta$-metilbutirato na redução do colesterol total e lipoproteína de baixa densidade também foram observados, porém, devido às poucas evidências, não há recomendações para tal finalidade. Nenhum efeito adverso da suplementação foi observado, sendo, aparentemente, segura, contudo a maioria dos estudos foi realizada em curto prazo (menos de 4 semanas) e em indivíduos destreinados. Assim, mais estudos são necessários para esclarecer o mecanismo fisiológico por meio do qual o $\beta$-hidroxi- $\beta$-metilbutirato exerce seus efeitos anticatabólicos em destreinados e para observar possíveis efeitos adversos.

Termos de indexação: Dieta. Hipertrofia. Suplementos dietéticos.

\footnotetext{
1 Universidade Gama Filho, Centro de Pesquisas Interdisciplinares em Saúde e Programa de Pós-Graduação em Educação Física. Rio de Janeiro, RJ, Brasil.

2 Universidade Gama Filho, Departamento de Nutrição. R. Manoel Vitorino, 625, Piedade, 20748-900, Rio de Janeiro, RJ, Brasil. Correspondência para/Correspondence to: C.M. MEIRELLES. E-mail: <claudiameirelles@yahoo.com.br>.

${ }^{3}$ Instituto M etodista Bennett, Departamento de N utrição. Rio de Janeiro, RJ, Brasil.
} 


\section{A B S T R A C T}

$\beta$-hydroxy- $\beta$-methylbutyrate is a leucine metabolite studied for its anticatabolic effects and potential benefits on strength and muscle mass gains associated with resistance training. The aim of this review was to discuss the effects of $\beta$-hydroxy- $\beta$-methylbutyrate supplementation on strength and hypertrophy in previously trained or untrained young and older adults. Studies undertaken with individuals initiating a resistance training program have suggested that a daily $\beta$-hydroxy- $\beta$-methylbutyrate supplementation dosage of 1.5 to $3.0 \mathrm{~g}$ may be responsive as an ergogenic aid up to the fourth or fifth week of resistance training. Such effects do not seem to remain afterward, when gains in strength and muscle mass are the result of resistance training only. In resistance trained subjects $\beta$-hydroxy- $\beta$-methylbutyrate supplementation did not show any additional benefits during resistance training. $\beta$-hydroxy- $\beta$-methylbutyrate supplementation has been shown to reduce total cholesterol and low-density lipoprotein cholesterol, although there seems to be no available recommendation for its supplementation, since scientific evidence is lacking. No $\beta$-hydroxy- $\beta$-methylbutyrate adverse effects have been observed, and its supplementation is apparently safe, however, most studies were conducted during a short-term period (less then 4 weeks) and with untrained individuals. There is a need for more studies to elucidate the physiological mechanisms underlying the anticatabolic action of $\beta$-hydroxy- $\beta$-methylbutyrate supplementation in untrained subjects, and to investigate possible adverse effects.

Indexing terms: Diet. Hypertrophy. Dietary supplements.

\section{N T R O D U ÇÃ O}

Muitos atletas e esportistas vivem em busca de meios que possam acelerar os ganhos de desempenho físico proporcionados pelo treinamento. Para isto, algumas estratégias podem ser sugeridas: dieta adequada e utilização de recursos ergogênicos nutricionais (termo aqui utilizado como sinônimo de suplementos nutricionais). Em academias de ginástica, o consumo de suplementos é freqüente. Pereira et al. ${ }^{1}$ observaram que 24\% dos 309 usuários de 7 academias de São Paulo consumiam, pelo menos, um tipo de suplemento, sendo que $90 \%$ o faziam diariamente. Os concentrados protéicos e aminoácidos foram consumidos por $39 \%$ da amostra, composta, em sua maioria, por praticantes de treinamento contra-resistência.

O American College of Sports Medicine $(\mathrm{ACSM})^{2}$ reconhece o treinamento contra-resistência (também conhecido como musculação) como passível de reduzir fatores de risco associados à doença coronariana, ao diabetes e ao câncer de cólon, assim como prevenir a osteoporose e ser coadjuvante no controle de peso e na preservação da capacidade funcional de idosos.

Embora no idoso o interesse principal que os leva a procurar essa atividade seja a promoção da saúde, entre adultos, o principal motivo parece ser a busca por ganhos de força e massa muscular ${ }^{3}$. Simultaneamente, cresce a busca por substâncias que prometem proporcionar benefícios estéticos. No entanto, apesar da crescente procura e utilização, muitas questões importantes precisam ser respondidas sobre o consumo de suplementos alimentares.

Em meta-análise, Nissen \& Sharp ${ }^{4}$ encontraram na literatura registro de mais de 200 suplementos alimentares que prometiam aumento da massa livre de gordura e ganhos de força durante o treinamento contra-resistência. Daqueles, apenas seis ( $\beta$-hidroxi- $\beta$-metilbutirato (HMB), creatina, proteína, cromo, dehidroepiandrosterona e androstenediona), atenderam aos critérios de inclusão. Os autores concluíram que apenas as suplementações de creatina e HMB demonstraram efeitos sobre a otimização dos ganhos de força e massa muscular em indivíduos praticantes de treinamento contra-resistência.

Os primeiros estudos com HM B, realizados em animais, apontaram efeitos desta substância sobre o metabolismo muscular, predominantemente, redução da proteólise ${ }^{5}$. No entanto, resultados controversos foram encontrados em estudos com seres humanos destreinados ou previamente treinados, examinando os efeitos da suple- 
mentação oral de HM B sobre as adaptações do músculo esquelético com o treinamento contra-resistência.

O objetivo da presente revisão de literatura foi discutir os possíveis efeitos da suplementação de HMB sobre a força e a hipertrofia de adultos jovens e idosos, iniciantes ou treinados, em exercícios contra-resistência.

A busca dos artigos científicos foi baseada em uma extensa procura na base de dados do M edline (1966-2005), além de material científico cedido pelo autor Steven Nissen (1990-1997). Referências listadas em revisões da literatura sobre 0 assunto, assim como outras retiradas de artigos originais, também foram pesquisadas. Para a recuperação de informação do Medline foram utilizadas as seguintes palavras-chave individualmente ou combinadas: beta-hydroxy-beta-methylbutyrate, HM B, leucine, supplementation, ergogenics. Estas palavras-chave foram associadas com exercise, resistance exercise, resistance training, strength training. A maior parte dos estudos encontrados estava em língua inglesa, embora artigos em português e espanhol também tenham sido considerados nesta procura. Todos os estudos relacionados aos efeitos da suplementação de HMB sobre o desempenho físico e os indicadores de saúde foram considerados, não importando os aspectos metodológicos, embora uma avaliação crítica fosse incluída no texto, quando necessário.

\section{O META BOLISMO DO HM B}

Estudos feitos em animais mostraram que o HM B é sintetizado, primeiramente, a partir do $\alpha$-cetoisocaproato (KIC) no fígado, ou seja, um subproduto do metabolismo da leucina. Aproximadamente $5 \%$ da leucina oxidada é convertida em $\mathrm{HM} \mathrm{B}^{6}$, além de ser produzida endogenamente no fígado de animais e humanos ${ }^{7}$. O HMB pode ser encontrado nos alimentos de origem animal e vegetal, como, por exemplo alfafa, toranja, peixe bagre e até mesmo no leite materno, estando também disponível comercialmente como um suplemento nutricional.
Especulou-se que o aminoácido de cadeia ramificada leucina e seus metabólitos poderiam estar envolvidos na modulação do sistema imune em animais, além de regular o metabolismo protéico ${ }^{6,8}$. Contudo, o que tem sido observado é que apenas um de seus metabólitos, o HMB, seria 0 responsável pelos efeitos positivos sobre o metabolismo protéico ${ }^{5,9,10}$.

A teoria por trás da suplementação é que, no citosol dos hepatócitos e das células musculares, o HMB é primeiro convertido em $\beta$-hidroxi- $\beta$-metilglutaril coenzima A (HM G-CoA) e pode seguir dois caminhos metabólicos distintos. O primeiro ocorre por meio da ação de uma enzima chamada HM G-COA redutase, a qual converte HM G-CoA em colesterol. O segundo ocorre por meio da enzima HM G-CoA sintetase que converte HMG-CoA em acetil-CoA, que é um substrato para geração de energia (Figura 1).

Quando há grande demanda para a síntese de colesterol, tal como ocorre nos períodos de rápido crescimento celular ou reconstituição de membranas, o HM G-CoA pode estar limitado. As células musculares estressadas ou danificadas não são capazes de produzir quantidades suficientes

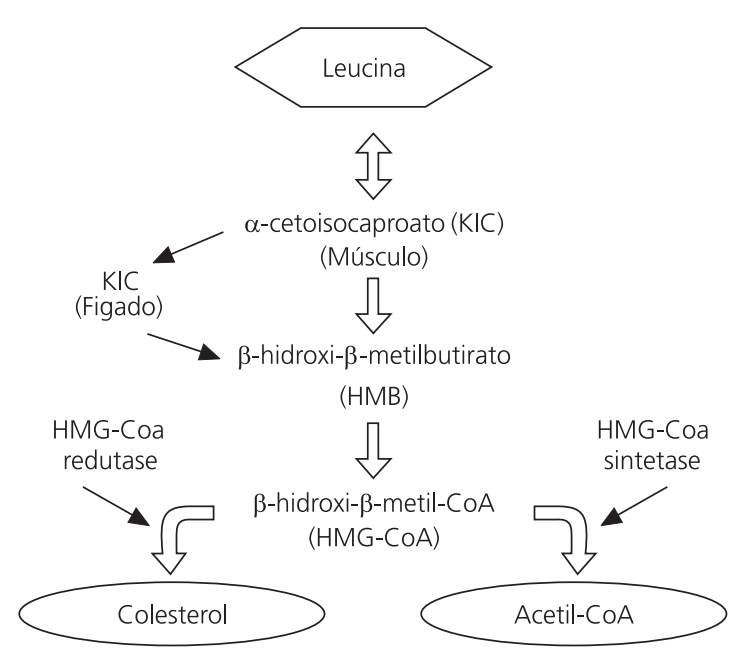

Figura 1. Visão geral do metabolismo de HM B a partir da leucina e $\alpha$-cetoisocaproato (KIC) em humanos. Adaptado de Nissen SL, Abumrad NN. Nutritional role of the leucine metabolite $\beta$-hydroxy $\beta$-methylbutyrate (HMB).

Fonte: J Nutr Biochem 1997; 8(6):300-311, com permissão de Elsevier. 
de HMG-CoA, comprometendo a síntese de colesterol adequada para as funções celulares ${ }^{7}$. Desse modo, o HMB poderia fornecer a quantidade necessária de HM G-CoA para a síntese de colesterol e a subseqüente reparação de membrana durante os períodos de estresse muscular aumentado.

De acordo com revisão de literatura de Slater \& Jenkins ${ }^{11}$, há especulações de que o HM B possa regular o metabolismo protéico, por desempenhar algum efeito sobre receptores celulares de cortisol, testosterona, hormônio do crescimento, fator de crescimento semelhante à insulina (IGF-1) e insulina, ou pela modulação de enzimas responsáveis pelo catabolismo do tecido muscular. Apesar disso, a suplementação de HM B não mostrou alteração nas concentrações plasmáticas de cortisol, testosterona, IGF-1 ou insulina. Os efeitos da suplementação de HM B em receptores hormonais, atividade específica de proteases e respostas de fase aguda não foram diretamente avaliados.

De qualquer forma, parece que o maior benefício do HMB reside na redução do catabolismo protéico, o que, conseqüentemente, resultaria em ganhos maiores no volume muscular e na força quando combinado com treinamento contra-resistência. Até o momento, no entanto, nenhum mecanismo fisiológico foi completamente elucidado a respeito dos efeitos do HMB sobre a proteólise.

Conforme o mencionado, o HMB é um precursor de colesterol via HM G-CoA. Assim, pode-se sugerir que as modificações na síntese de colesterol poderiam produzir alterações nas concentrações de colesterol sangüíneo.

Nissen et al. ${ }^{7}$ reuniram nove estudos com duração de três a oito semanas, nos quais homens e mulheres jovens e idosos, praticantes ou não de treinamento contra-resistência, foram suplementados com $3 \mathrm{~g}$ de HM B por dia. Observou-se que sujeitos com concentrações sangüíneas elevadas de colesterol total $(>200 \mathrm{mg} / \mathrm{dL})$, suplementados com HMB, apresentaram uma redução de 5,8\% $(p<0,05)$, comparados com o grupo não suplementado. 0 mesmo não aconteceu com sujeitos que tinham menores concentrações de colesterol total $(<200 \mathrm{mg} / \mathrm{dL})$, nos quais a redução não foi significante. Isso sugere que o HMB seria mais efetivo em reduzir o colesterol quando suas concentrações sanguíneas se encontram acima de $200 \mathrm{mg} / \mathrm{dL}$, o que se associaria com risco aumentado de doenças cardiovasculares.

Há poucos estudos disponíveis sobre os efeitos do HMB sobre a lipidemia. A limitada informação sobre 0 efeito hipolipêmico deste suplemento provém de observações de Nissen e seus colaboradores, as quais nunca foram confirmadas por outros pesquisadores. 0 estudo feito por Gallagher et al. ${ }^{12}$, com duração de oito semanas, incluindo homens destreinados participando de um programa de treinamento contra-resistência, não apóia tais achados, uma vez que nenhuma diferença significativa foi observada na colesterolemia após a suplementação e o treinamento.

Embora seja claro que o HMB possa ser convertido em colesterol, o mecanismo por meio do qual sua suplementação foi capaz de reduzir as concentrações sangüíneas não é conhecido. M ais estudos são necessários para esclarecer esta indagação.

À luz da literatura atual, a suplementação de HMB parece não oferecer efeitos negativos. Parâmetros tais quais indicadores de função hepática, renal e hematológica não parecem ser alterados com o consumo diário de até $6 \mathrm{~g}$ de $\mathrm{HMB}^{12} \mathrm{e}$ - Comitê Olímpico Internacional o classifica como uma substância legal.

\section{EFEITOS DO HMB SOBRE FORÇA E HIPERTROFIA}

Os primeiros estudos sobre os efeitos do HM B sobre o metabolismo muscular foram feitos com animais. Ostaszewksi et al. ${ }^{5}$ examinaram as taxas de proteólise e síntese protéica em ratos e galinhas e observaram reduções médias de, aproximadamente, $18 \%(p<0,05)$ nas taxas de proteólise em fibras musculares cultivadas com HM B a 1mM , 
em ambos os animais. A síntese protéica foi aumentada em, aproximadamente, $6 \%$, porém de forma não significante.

\section{Efeitos do HMB em indivíduos iniciando o programa de treinamento contra-resistência}

Alguns estudos demonstraram que suplementação de HMB durante o treinamento contra-resistência resulta em maior crescimento muscular e concomitante aumento na força em sujeitos destreinad os ${ }^{13-16}$.

Panton et al. ${ }^{17}$ e Knitter et al. ${ }^{18}$ reportaram redução nas concentrações plasmáticas de creatina cinase (CK) e lactato desidrogenase ( $L D H)$, que são indicadores de danos na membrana muscular, assim como diminuição da excreção urinária de 3-methil-histidina (3-M H) durante o treinamento, o que indica menores taxas de proteólise.

Parece que o HMB sem a presença do treinamento contra-resistência não influencia as mudanças na composição corporal. Quando um grupo de 37 mulheres sedentárias foi suplementado com placebo ou HM B (3g/dia) durante quatro semanas, nenhuma mudança significativa foi observada na composição corporal. Quando esta mesma dosagem foi repetida após quatro semanas de treinamento contra-resistência, 3x/semana (não detalhado), os ganhos de força em músculos peitorais e membros superiores avaliados por exercício em supino foram $7 \%$ maiores e os de massa livre de gordura, avaliada por pesagem hidrostática, foram $0,48 \mathrm{~kg}$ maiores no grupo HMB do que no placebo $(p<0,05)^{19}$.

A intensidade do treinamento contra-resistência é comumente determinada pelo número máximo de repetições de um movimento a um determinado percentual de uma repetição máxima (1 RM ). Assim, um exercício muito intenso pode ser alcançado com muitas repetições a um baixo percentual de 1 RM ou com poucas repetições a um percentual alto de 1 RM . A carga para 1 RM é operacionalmente definida como a maior carga possível de ser levantada, em uma determinada amplitude de movimento uma única vez, e realizada de maneira correta.

Nissen et al. ${ }^{14}$ submeteram homens destreinados a 2 experimentos. No primeiro, com 3 semanas de duração, os sujeitos foram divididos em 6 grupos, quais sejam, (1) placebo, (2) 1,5g HM B, (3) 3,0g HM B, (4) suplemento hiperprotéico (37g de proteína e $270 \mathrm{kcal})$ ), suplemento hiperprotéico (5) mais $1,5 \mathrm{~g} \mathrm{HMB}$ ou (6) suplemento hiperprotéico mais $3,0 \mathrm{~g} \mathrm{HMB}$. Todos realizavam um programa de treinamento contra-resistência consistindo de 3 séries de 3-5 repetições a 90\% de 1 RM, 3 vezes por semana. No segundo experimento, com 7 semanas de duração, os sujeitos foram suplementados diariamente com placebo ou $3,0 \mathrm{~g}$ de HM B e realizaram treinamento contra-resistência por 2 a 3 horas por dia, 6 dias por semana (não detalhado). 0 maior achado desse estudo foi que no primeiro experimento, a suplementação diária com 1,5 ou 3,0g de HM B reduziu a proteólise e o dano muscular induzido pelo exercício. Foram observadas reduções de $20 \%$ na excreção urinária de 3-MH e 20\%-60\% nas concentrações plasmáticas de CK e LDH. Foram verificados, ainda, durante as 3 semanas iniciais, ganhos significantemente maiores de força total no grupo suplementado em comparação ao placebo $(13 \%$ com $1,5 \mathrm{~g}, 18,4 \%$ com $3,0 \mathrm{~g}$ e $8 \%$ no placebo; $p<0,02)$. Os ganhos de massa livre de gordura não foram estatisticamente diferentes entre os grupos e nenhum efeito adicional da suplementação de proteína foi observado.

No segundo experimento, o qual foi realizado durante um período maior (7 semanas), os sujeitos de ambos os grupos apresentaram ganhos similares de força, com exceção apenas para 0 supino, no qual o grupo HMB obteve maiores aumentos do que o placebo $(5,4$ versus - vs $15 \%$, respectivamente). Quanto à massa livre de gordura, os ganhos só foram significantemente maiores para o grupo HMB entre o 140 e 039 응 dia de protocolo $(p<0,05)$. Após, nenhuma diferença significativa foi observada na força e composição corporal entre os grupos ${ }^{14}$. 
Vale ressaltar que a utilização da impedância bioelétrica (BIA) para avaliação da composição corporal está condicionada a fontes de erro. A principal é o nível de hidratação do indivíduo ${ }^{20}$. Se este não for controlado, a interpretação dos resultados fica comprometida e, neste estudo, os autores não relataram cuidados especiais com 0 nível de hidratação dos sujeitos antes da avaliação com equipamento de BIA.

Além disso, diferentemente do primeiro experimento, no segundo, o consumo dietético não foi monitorado e apenas o grupo HM B recebeu o suplemento hiperprotéico. Os autores não revelaram se os horários de consumo do suplemento coincidiam com o treinamento contra-resistência, o que pode ter influenciado os resultados. É reconhecido que, dependendo do horário de consumo, uma mistura de carboidratos e proteína pode otimizar em até $400 \%$ as taxas de síntese protéica muscular quando ingerida em horários próximos ao de execução do exercício contra-resistência ${ }^{21}$.

Jówko et al. ${ }^{22}$ conduziram um estudo duplo-cego controlado e randomizado com três semanas de duração no qual indivíduos iniciando treinamento contra-resistência foram divididos em quatro grupos: placebo, creatina $(20 \mathrm{~g} /$ dia durante a 1 a semana e $10 \mathrm{~g} /$ dia nas duas seguintes), HMB (3g/dia) ou HM B + creatina. Ao final do período de treinamento contra-resistência e suplementação, todos os grupos ganharam força $(p<0,05)$, entretanto, os ganhos foram maiores no grupo HM B + creatina $(71,6 \mathrm{~kg})$, tendo sido de $57,3 \mathrm{~kg}$ no grupo creatina, 58,9 no HMB e $19,7 \mathrm{~kg}$ no placebo. Quanto à massa livre de gordura, avaliada por BIA, os sujeitos no grupo HM B + creatina também obtiveram ganhos significantemente maiores do que aqueles nos grupos que receberam apenas creatina ou HMB $(2,4 \mathrm{~kg}$ vs 1,8 e $1,2 \mathrm{~kg}$, respectivamente). 0 grupo HM B não demonstrou maiores ganhos de massa livre de gordura, quando comparado ao placebo. Adicionalmente, o grupo HMB e o grupo creatina + HMB apresentaram valores significantemente menores da enzima $\mathrm{CK}$ em comparação aos grupos placebo e creatina. Os autores concluíram que a creatina e o HMB podem desempenhar ação aditiva sobre os ganhos de força e massa livre de gordura.

Contudo, este estudo traz graves limitações na interpretação dos seus resultados. Os sujeitos que consumiram creatina e HMB + creatina aumentaram de forma significativa o conteúdo de água corporal total, o que compromete sobremaneira os resultados proporcionados pela BIA ${ }^{20}$. Sendo assim, os ganhos em massa livre de gordura podem ter sido devidos apenas ao aumento de hidratação celular proporcionada pela creatina, conforme é comumente observado no início da suplementação desta substância²3.

Outra limitação ocorreu no controle dietético, o grupo suplementado apenas com creatina reduziu em até $20 \%$ a ingestão energética, quando comparado aos grupos HMB. Isso pode ter prejudicado os ganhos potenciais, mascarando uma possível ação isolada da creatina como agente ergogênico e permitindo a detecção de melhores resultados no grupo HM B + creatina, 0 qual não limitou a ingestão energética.

A creatina tem mostrado efeitos ergogênicos tanto para os ganhos de força, quanto de massa livre de gordura em desenhos experimentais agudos e crônicos bem controlados. Esses efeitos vêm sendo observados de forma mais consistente do que os eventualmente verificados com o $\mathrm{HMB}^{23}$. Parece improvável que exista uma ação sinérgica entre ambos os suplementos. O'Connor $\&$ Crowe ${ }^{24}$ investigaram seus possíveis efeitos aditivos e não os constataram.

Gallagher et al. ${ }^{25}$, com um protocolo de duração de oito semanas de treinamento contra-resistência, no qual os sujeitos eram suplementados com $38 \mathrm{mg} / \mathrm{kg} / \mathrm{dia}$ ou $76 \mathrm{mg} / \mathrm{kg} / \mathrm{dia}$, evidenciaram ganhos de força não estatisticamente diferentes entre os grupos. Contudo, os aumentos de massa livre de gordura no grupo que consumiu $38 \mathrm{mg} / \mathrm{kg} / \mathrm{dia}$ de HM B foi significativamente maior do que os observados nos grupos placebo e $76 \mathrm{mg} / \mathrm{kg} / \mathrm{dia}(1,9 \mathrm{~kg}$ vs $0,0 \mathrm{vs}-0,2 \mathrm{~kg}$, respectivamente). Ressalta-se que a avaliação da composição corporal foi feita por meio da antropometria, 
o que leva a dúvidas na interpretação desse achado, dado o viés associado à tomada de dobras cutâneas ${ }^{26}$.

Este estudo trouxe uma importante contribuição, qual seja, a de que doses de HMB superiores a 3,0g/dia não favorecem o aumento de seus efeitos em indivíduos destreinados iniciando treinamento contra-resistência.

Estudos realizados com animais revelaram que o consumo de elevadas doses de HM B estão relacionadas a menores taxas de captação muscular. 0 tecido muscular de suínos somente absorve HMB quando este está presente em baixas concentrações sangüíneas ${ }^{27}$. Se ocorrer da mesma maneira em humanos, a suplementação de $76 \mathrm{mg} / \mathrm{kg} / \mathrm{dia}$ pode ter estado menos biodisponível e, por isso, não influenciou os ganhos de força e massa livre de gordura dos sujeitos.

Em relação aos efeitos do HMB sobre ganhos de força e possíveis mudanças na composição corporal em idosos ( $\geq 70$ anos), Vukovich et al. ${ }^{15}$, por meio de um desenho randomizado e duplo-cego, revelaram que a suplementação de $3,0 \mathrm{~g}$ de HM B, associada ao treinamento contra-resistência duas vezes por semana, ofereceu ganhos significativamente maiores de força e massa livre de gordura do que os observados no placebo nas quatro semanas iniciais. Ao final de oito semanas de treinamento e suplementação de HMB, embora os ganhos de força e a redução da gordura corporal tenham se mantido superiores no grupo HMB, as modificações na massa livre de gordura ocorreram de forma similar entre os grupos. Nenhuma interação gênero vs suplementação de HM B foi verificada. Uma crítica pertinente que se faz a este estudo é a ausência de detalhes sobre os testes de força e método de avaliação da composição corporal.

Vukovich et al. ${ }^{16}$ realizaram outro estudo com o objetivo de analisar o efeito da suplementação de HMB na composição corporal de idosos ( $\geq 70$ anos). Trinta e um homens e mulheres divididos em grupos HM B ( $3,0 \mathrm{~g}$ por dia) e placebo realizaram treinamento contra-resistência cinco vezes por semana, durante oito semanas. 0 grupo suplementado reduziu significativamente o percentual de gordura corporal total, em comparação ao grupo não suplementado. Contudo, não houve diferenças significantes nos ganhos de massa livre de gordura e força entre os grupos, embora os autores tenham considerado valores de $p=0,08$ como uma tendência de maiores ganhos no grupo HMB. Segundo os mesmos, a redução no percentual de gordura corporal pode ter sido devido ao aumento da massa livre de gordura e/ou à redução da massa de gordura.

Vale afirmar que a perda de gordura corporal só foi significativa quando estimada pela medida das dobras cutâneas. Quando estimada por absortometria radiológica de dupla energia (DEXA), que é um método mais acurado de avaliação da composição corporal, a redução não foi significativa.

Utilizando grupos da mesma faixa etária, Panton et al. ${ }^{28}$ não identificaram diferenças significantes nos valores médios de força ao final de oito semanas de treinamento contra-resistência (3 x 8-12 repetições a $75 \%-85 \%$ de 1 RM) entre os grupos HM B e placebo. Ambos ganharam força de forma similar (extensão de joelhos - HMB: $59,5 \mathrm{~kg}$; placebo: $60,5 \mathrm{~kg}$ e supino - HM B: $50,5 \mathrm{~kg}$; placebo: $51,8 \mathrm{~kg}$ ), o que indicou que os ganhos foram inerentes às adaptações ao treinamento e não puderam ser potencializados pela suplementação.

Em idosos, com exceção do estudo de Vukovich et al. ${ }^{15}$, a suplementação de HM B não parece ser capaz de otimizar os ganhos de força e massa livre de gordura proporcionados pelo treinamento contra-resistência.

Embora pesquisas adicionais sejam necessárias, grande parte dos estudos disponíveis sugere que a suplementação de $3,0 \mathrm{~g} / \mathrm{dia}$ de HM B pode favorecer os ganhos de força e hipertrofia muscular em sujeitos jovens destreinados, iniciando um programa de treinamento contra-resistência. Contudo, tais efeitos parecem não se manter significativos após o primeiro mês de treinamento contra-resistência e suplementação. 
Uma limitação na interpretação dos resultados dos estudos com HM B, entretanto, refere-se aos marcadores de catabolismo utilizados. Em vários estudos, a 3-MH e a CK foram dosadas. Estes são indicadores pouco precisos da degradação de actina e miosina no músculo esquelético. As concentrações de 3-MH também aumentam em resposta ao catabolismo da musculatura lisa ${ }^{29}$ e proteínas citosólicas como a $\mathrm{CK}$, que também não reflete apenas a extensão do dano estrutural da célula muscular. Sugere-se a avaliação de proteínas estruturais, tais como cadeia pesada da miosina e troponina ${ }^{30}$.

O Quadro 1 resume os resultados de estudos desenvolvidos para investigar os efeitos da suplementação de HMB sobre variáveis bioquímicas, força e composição corporal em indivíduos iniciando o programa de treinamento contra-resistência.

Quadro 1. Resumo de estudos abordando os efeitos da suplementação de HMB em indivíduos iniciando o programa de treinamento contra-resistência.

\begin{tabular}{|c|c|c|c|c|c|c|}
\hline Autor & Amostra & Duração & Suplementação & Bioquímica & Força & Composição corporal \\
\hline $\begin{array}{l}\text { Nissen } \\
\text { et al. }{ }^{13}\end{array}$ & $\begin{array}{l}23 \mathrm{H} \\
\text { adultos jovens }\end{array}$ & 4 SEM & PLA ou $3,0 \mathrm{~g} \mathrm{HMB/dia.}$ & NA & MMSS: HMB>PLA & $\begin{array}{l}\text { MM: HMB>PLA } \\
\text { MG: HMB<PLA }\end{array}$ \\
\hline \multirow[t]{2}{*}{$\begin{array}{l}\text { Nissen } \\
\text { et al. }{ }^{14}\end{array}$} & Estudo 1: $41 \mathrm{H}$ & $\begin{array}{l}\text { Estudo 1: } \\
3 \text { SEM }\end{array}$ & $\begin{array}{l}\text { Estudo 1: seis condições; } \\
\text { (1) PLA; (2) 1,5; (3) 3,0g } \\
\text { HMB/dia; (4) SHP; (5) SHP } \\
\text { + 1,5; (6) SHP + 3,0g } \\
\text { HMB/dia }\end{array}$ & $\begin{array}{l}\text { Estudo } 1: \\
\text { 3-M H: HMB<PLA } \\
\text { até a 2 }{ }^{\text {a SEM }}\end{array}$ & $\begin{array}{l}\text { Estudo } 1 \text { : } \\
\text { M M SS:HM B=PLA } \\
\text { M M II: HM B>PLA } \\
\text { Total: HM B>PLA }\end{array}$ & $\begin{array}{l}\text { Estudo } 1 \text { : } \\
\text { MM: HM B=PLA } \\
\text { MG: HMB=PLA } \\
\text { MCT: HM B>PLA }\end{array}$ \\
\hline & $\begin{array}{l}\text { Estudo } 2: 28 \mathrm{H} \\
\text { adultos jovens }\end{array}$ & $\begin{array}{l}\text { Estudo 2: } \\
7 \text { SEM }\end{array}$ & $\begin{array}{l}\text { Estudo 2: duas condições; } \\
\text { (1) PLA; (2) 3,0g HM B/dia + } \\
\text { SHP }\end{array}$ & Estudo 2: NA & $\begin{array}{l}\text { Estudo 2: } \\
\text { MMSS: HMB>PLA }\end{array}$ & 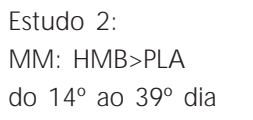 \\
\hline $\begin{array}{l}\text { Vukovich } \\
\text { et al. }{ }^{15}\end{array}$ & $\begin{array}{l}15 \mathrm{H} \text { e } 16 \mathrm{M} \\
\text { idosos }\end{array}$ & 8 SEM & PLA ou $3,0 \mathrm{~g} \mathrm{HMB/dia}$ & NA & $\begin{array}{l}\text { Flexão joelhos: } \\
\text { HM B>PLA } \\
\text { MM II: HM B>PLA } \\
\text { até SEM } 4\end{array}$ & $\begin{array}{l}\text { MM: } \mathrm{HMB}=\mathrm{PLA} \\
\text { MG: } \mathrm{HMB}<\mathrm{PLA}\end{array}$ \\
\hline $\begin{array}{l}\text { Vukovich } \\
\text { et al. }{ }^{16}\end{array}$ & $\begin{array}{l}15 \mathrm{H} \text { e } 16 \mathrm{M} \\
\text { idosos }\end{array}$ & 8 SEM & PLA ou $3,0 \mathrm{~g} \mathrm{HMB/dia.}$ & NA & $\begin{array}{l}\text { Exercício no } \\
\text { puxador e força } \\
\text { total: HMB>PLA } \\
\text { Flexão de joelhos: } \\
\text { HMB>PLA após as } \\
\text { SEM } 4 \text { e } 8\end{array}$ & $\begin{array}{l}\text { M M : HMB=PLA } \\
\text { MG: } H M B<P L A\end{array}$ \\
\hline $\begin{array}{l}\text { Nissen } \\
\text { et al. }{ }^{19}\end{array}$ & $\begin{array}{l}37 \mathrm{M} \\
\text { adultos jovens }\end{array}$ & 4 SEM & PLA ou $3,0 \mathrm{~g} \mathrm{HMB/dia.}$ & NA & MMSS: HMB>PLA & $\begin{array}{l}\text { MM: HMB>PLA } \\
\text { MG: } H M B=P L A\end{array}$ \\
\hline $\begin{array}{l}\text { Jówko } \\
\text { et al. }{ }^{22}\end{array}$ & $\begin{array}{l}40 \mathrm{H} \\
\text { adultos jovens }\end{array}$ & 3 SEM & $\begin{array}{l}\text { Quatro condições: (1) PLA, } \\
\text { (2) CR (20g de CR/dia por } \\
7 \text { dias; após, } 10 \mathrm{~g} \text { de CR/ } \\
\text { dia por } 14 \text { dias), (3) HMB } \\
\text { (3,0g HM B/dia) ou (4) CR/ } \\
\text { HMB (3,0g HM B/dia + 20g } \\
\text { de CR/dia por } 7 \text { dias; após, } \\
10 \mathrm{~g} \text { de CR/dia por } 14 \text { dias). }\end{array}$ & $\begin{array}{l}\text { CK: HM B<PLA, } \\
\text { CR e CR/HMB }\end{array}$ & $\begin{array}{l}\text { Total: HMB, } \\
\text { CR>PLA e CR/HM B }\end{array}$ & $\begin{array}{l}\text { MCT: HMB e CR>PLA } \\
\text { e CR/HMB } \\
\text { MM: CR>HMB, PLA } \\
\text { e CR/HMB } \\
\text { MG: HMB=PLA, CR e } \\
\text { CR/HM B }\end{array}$ \\
\hline $\begin{array}{l}\text { Gallagher } \\
\text { et al. }{ }^{25}\end{array}$ & $\begin{array}{l}37 \mathrm{H} \\
\text { adultos jovens }\end{array}$ & 8 SEM & PLA; 3,0 ou $6,0 \mathrm{~g} \mathrm{HMB/dia}$ & $\begin{array}{l}\text { CK: HMB<PLA até } \\
48 h \text { após TCR }\end{array}$ & $\begin{array}{l}1 \mathrm{RM}: \mathrm{HMB}=\mathrm{PLA} \\
\text { Total: } \mathrm{HMB}=\mathrm{PLA}\end{array}$ & $\begin{array}{l}\text { MM: HMB } 3 g>P L A \text { e } \\
\text { HMB } 6 g \\
\text { MG: HMB=PLA }\end{array}$ \\
\hline
\end{tabular}

$<$ e >: valores estatisticamente significativos $(p<0,05)$ entre os grupos; TCR: treinamento contra-resistência; H: homens; $M$ : mulheres; PLA: placebo; SHP: suplemento hiperprotéico; CK: creatina cinase; 3-M H: 3-metil-histidina; M M : massa magra; M G: gordura corporal; M M SS: membro superior; MM II: membro inferior; MCT: massa corporal total; SCP: suplemento carboidrato/proteína; CR: creatina; HMB: beta-hidroxi-betametilbutirato; SEM : semana; NA: não analisado; Todos os estudos foram randomizados e duplo-cegos. 


\section{Efeitos do HMB em indivíduos treinados em atividade contra-resistência}

Estudos com indivíduos destreinados iniciando um programa de treinamento contra-resistência sugerem que suplementação diária de HMB (3g) pode favorecer o aumento da força e massa muscular. Contudo, parece que indivíduos treinados não respondem da mesma maneira que indivíduos destreinados.

Ransone et al. ${ }^{31}$ conduziram um estudo randomizado e duplo-cego de quatro semanas de duração com jogadores de futebol americano. Cada sujeito participou de sessões de treinamento contra-resistência quatro vezes na semana, executando 10 exercícios com 8-12 séries de 2-10 repetições por exercício a 70\%-90\% de 1 RM por sessão. No final do estudo, nenhuma modificação significativa na força e na composição corporal ocorreu entre os grupos (placebo e $3 \mathrm{~g}$ de HM B). Segundo os autores, a falta de diferenças significativas pode ser, em parte, explicada pela baixa ingestão energética dos indivíduos durante o treinamento contra-resistência, pois o consumo dietético não foi controlado, assim como pelo maior volume total de treinamento neste estudo, em comparação a outros sobre HMB, uma vez que os atletas em questão associavam exercícios aeróbios à rotina semanal de treinamento.

Kreider et al. ${ }^{32}$ reuniram 41 jogadores de futebol americano com o objetivo de analisar os efeitos da suplementação de HMB com ou sem creatina na composição corporal. Os sujeitos foram divididos em três grupos: placebo, HM B (3g/dia) ou creatina + HMB $(15,75 \mathrm{~g} /$ dia de creatina $+3 \mathrm{~g} /$ dia de HMB). 0 estudo teve duração de 28 dias e nesse período os sujeitos realizaram treinamento contra-resistência cinco horas por semana. A massa livre de gordura só foi significativamente aumentada no grupo creatina + HMB e nenhuma diferença foi observada no percentual de gordura corporal entre os grupos. Os resultad os indicaram que a suplementação de HMB não afetou as mudanças na composição corporal em atletas de alto nível, mas, aparentemente, a presença da creatina foi decisiva para os resultados eviden- ciados. Neste estudo, teria sido de valia para a interpretação dos resultados se houvesse também um grupo creatina, para evitar qualquer dúvida a respeito de um possível efeito aditivo creatina + HM B, tal como sugerido por Jówko et al. ${ }^{21}$.

Kreider et al. ${ }^{33}$ conduziram um estudo duplo-cego e randomizado; durante 28 dias, jogadores de futebol americano foram divididos em dois grupos recebendo diariamente placebo ou $3 \mathrm{~g}$ de HM B e submetidos a treinamento contra-resistência consistindo de 1-3 séries com 2-8 repetições a $60-95 \%$ de 1 RM, três horas por semana em cicloergômetro e 12 tiros de seis segundos (esforço máximo) com 30 segundos de recuperação. Nenhuma diferença significativa foi observada em marcadores de catabolismo (CK e LDH), composição corporal e desempenho da força e velocidade de corrida entre os grupos.

Em um estudo randomizado e duplo-cego, Slater et al. ${ }^{34}$ avaliaram, durante seis semanas, possíveis mudanças em força e composição corporal de atletas de pólo aquático e remo divididos nos grupos placebo e $3,0 \mathrm{~g}$ de HMB. Todos os sujeitos participaram de um programa de treinamento contra-resistência 2-3 vezes por semana, constando de 3-5 séries com 4-6 repetições. A composição corporal foi avaliada por DEXA e o controle dietético por meio de registro alimentar de três dias. Além disso, para garantir um adequado aporte energético, todos os sujeitos foram solicitados a ingerir diariamente, junto com a sua dieta habitual, um suplemento nutricional (280 calorias, $24 \mathrm{~g}$ carboidrato, $42 \mathrm{~g}$ proteína, $2 \mathrm{~g}$ gordura). A força, a massa corporal total e a massa magra aumentaram significativamente em ambos os grupos. Sendo assim, a administração de HM B não influenciou as mudanças proporcionadas pelo treinamento contra-resistência.

Thomson ${ }^{35}$, a partir de um desenho duplo-cego e randomizado, submeteu 34 sujeitos treinados a treinamento contra-resistência durante nove semanas. Parte dos sujeitos recebeu placebo e parte, $3 \mathrm{~g}$ de HMB. 0 controle dietético foi feito por meio de registros de três dias. Nenhuma mudança significativa foi encontrada nas medidas 
antropométricas entre os grupos. No entanto, 0 grupo HMB apresentou ganhos significativos de força na extensão de joelhos, comparado ao grupo placebo. A pós analisar os registros, 0 autor observou que o grupo HMB consumiu dietas com maiores porcentagens de energia na forma de carboidratos e menores na forma de gordura do que o grupo placebo.

A presença de maiores teores de carboidrato pode ter influenciado os resultados, uma vez que o consumo deste nutriente logo após o exercício contra-resistência exerce efeitos importantes no balanço protéico, aumentando a produção e a secreção de insulina, o que reduz a degradação protéica muscular e produz um balanço protéico mais positivo pós-exercício contra-resistência, conforme o descrito em revisão de literatura de $\mathrm{M}$ eirelles $^{21}$.

Panton et al. ${ }^{28}$ estudaram homens e mulheres treinados e destreinados executando treinamento contra-resistência durante quatro semanas. Todos os sujeitos foram separados em grupos segundo gênero. Cada grupo recebeu diariamente placebo ou HM B (3g). Todos os sujeitos treinaram três vezes na semana durante quatro semanas. Apenas os homens suplementados com HMB demonstraram, ao final do estudo, melhora significativa da força muscular de membros superiores, comparados com o grupo placebo. Nenhuma interação significativa tempo vs grupo foi observada nas variáveis relativas à composição corporal. Os maiores achados deste estudo foram que a resposta ao HMB não dependeu do nível de treinamento e que homens responderam melhor à suplementação de HMB que mulheres.

De acordo com esses resultados, a suplementação de HMB poderia potencializar as mudanças associadas ao treinamento contra-resistência somente em alguns grupos musculares e apenas em homens, independentemente do nível de treinamento. Embora ainda não se conheçam as razões que explicariam o possível favorecimento decorrente do HMB em determinados grupos musculares, em detrimento de outros, os autores tentaram explicar os achados por meio da hipótese de maior disponibilidade de HM G-CoA como precursor da síntese de colesterol para reparação de membranas celulares microlesionadas pelo treinamento. Contudo, as concentrações de CK não foram significativamente diferentes entre as condições pré-pós treinamento contra-resistência nos grupos placebo e HMB, o que dificulta a confirmação dessa possibilidade. Além disso, a duração do estudo foi muito curta para possibilitar maiores inferências sobre os efeitos crônicos do HMB.

Não se conhece o motivo responsável pelos diferentes resultados, observados em sujeitos treinados em relação aos destreinados. Poderia se especular, no entanto, que estaria relacionado às menores respostas catabólicas ao exercício contra-resistência observadas em indivíduos treinados ${ }^{36}$.

O Quadro 2 resume os resultados de estudos desenvolvidos para investigar os efeitos da suplementação de HM B sobre variáveis bioquímicas, força e composição corporal em indivíduos treinados em exercício contra-resistência.

\section{O N C L U S Ã O}

A presente revisão apontou que a suplementação de HMB em indivíduos destreinados submetidos a programas de treinamento contra-resistência pode levar a efeitos ergogênicos sobre força e hipertrofia. Porém, os ganhos parecem ser significativamente maiores entre os suplementados somente no início do programa. Após as primeiras semanas de treinamento contra-resistência, os resultados são similares, tanto em sujeitos suplementados quanto não suplementados.

As evidências provenientes de estudos com indivíduos já treinados em atividade contra-resistência são limitadas, mas as disponíveis apontam que o HM B parece não exercer efeitos ergogênicos sobre os ganhos de força e massa livre de gordura neste grupo. 
Quadro 2. Resumo de estudos abordando os efeitos da suplementação de HMB em indivíduos treinados em um programa de treinamento contra-resistência.

\begin{tabular}{|c|c|c|c|c|c|c|}
\hline Autor & Amostra & Duração & Suplementação & Bioquímica & Força & Composição corporal \\
\hline $\begin{array}{l}\text { Panton } \\
\text { et al. }{ }^{17}\end{array}$ & $\begin{array}{c}39 \mathrm{H} \text { e } 36 \mathrm{M} \\
\text { treinados e } \\
\text { destreinados }\end{array}$ & 4 SEM & PLA ou $3,0 \mathrm{~g} \mathrm{HMB/dia}$ & $\begin{array}{l}\text { CK: HMB=PLA em } \\
\mathrm{H} \text { e M }\end{array}$ & $\begin{array}{l}\text { M M SS: HM B>PLA (H) } \\
\text { M M SS: HM B=PLA (M) }\end{array}$ & $\begin{array}{l}\text { M M: HM B=PLA }(H \text { e } M) \\
M G: H M B=P L A(H \text { e } M)\end{array}$ \\
\hline $\begin{array}{l}\text { Ransone } \\
\text { et al. }{ }^{31}\end{array}$ & $35 \mathrm{H}$ & 4 SEM & PLA ou $3,0 \mathrm{~g} \mathrm{HMB} / \mathrm{dia}$ & NA & Total: HM B=PLA & $\begin{array}{l}\text { MM: } H M B=P L A \\
\text { MG: } H M B=P L A \\
\text { MCT: } H M B=P L A\end{array}$ \\
\hline $\begin{array}{l}\text { Kreider } \\
\text { et al. }{ }^{32}\end{array}$ & $41 \mathrm{H}$ & 28 dias & $\begin{array}{l}\text { Três condições: (1) PLA; (2) } \\
\text { HMB (3,0 /dia); (3) } \\
\text { HM B/CR (3,0g HMB/dia } \\
+15,75 \text { g CR/dia) }\end{array}$ & NA & NA & $\begin{array}{l}\text { MCT: HMB=PLA e } \\
\text { HMB/CR } \\
\text { M M: HMB } \\
\text { CR>HMB e PLA } \\
\text { MG: HMB }=P L A \text { e }\end{array}$ \\
\hline $\begin{array}{l}\text { Kreider } \\
\text { et al. }{ }^{34}\end{array}$ & $28 \mathrm{H}$ & 28 dias & PLA ou $3,0 \mathrm{~g} \mathrm{HMB/dia}$ & $\begin{array}{l}\text { CK: HMB=PLA } \\
\text { LDH: HM B=PLA }\end{array}$ & $\begin{array}{l}\text { Desempenho no } \\
\text { sprint: } \mathrm{HMB} B=\mathrm{PLA}\end{array}$ & $\begin{array}{l}\mathrm{HM} \mathrm{B} / \mathrm{CR} \\
\text { M CT: HM B=PLA } \\
\text { M M : HM B=PLA }\end{array}$ \\
\hline Thomson ${ }^{35}$ & $34 \mathrm{H}$ & 9 SEM & PLA ou $3,0 \mathrm{~g} \mathrm{HMB/dia}$ & NA & $\begin{array}{l}\text { Extensão de joelhos: } \\
\text { HM B>PLA }\end{array}$ & $\begin{array}{l}\text { MG: } H M B=P L A \\
M M: \quad H M B=P L A\end{array}$ \\
\hline $\begin{array}{l}\text { Kreider } \\
\text { et al. }{ }^{37}\end{array}$ & $40 \mathrm{H}$ & 4 SEM & $\begin{array}{l}\text { PLA, } 3,0 \text { ou } 6,0 \mathrm{~g} \mathrm{HMB} / \mathrm{dia} \\
+ \text { SCP nos três grupos. }\end{array}$ & $\begin{array}{l}\text { CK: HM B=PLA } \\
\text { LDH: HM B=PLA }\end{array}$ & Total: $\mathrm{HMB} B=\mathrm{PLA}$ & $\begin{array}{l}\text { MG: } \quad H M B=P L A \\
M M: \quad H M B=P L A\end{array}$ \\
\hline $\begin{array}{l}\text { Hoffman } \\
\text { et al. }{ }^{38}\end{array}$ & $26 \mathrm{H}$ & 10 dias & PLA ou $3,0 \mathrm{~g} \mathrm{HMB/dia}$ & $\begin{array}{l}\text { CK: HM B=PLA } \\
\text { M ioglobina: } \\
\text { HM B=PLA } \\
\text { Cortisol:HM B=PLA } \\
\text { Testosterona: } \\
\text { HM B=PLA }\end{array}$ & $\begin{array}{l}\text { Desempenho no } \\
\text { sprint:HM B=PLA }\end{array}$ & $\begin{array}{l}\text { MG: HMB=PLA } \\
\text { MCT: HM B=PLA }\end{array}$ \\
\hline
\end{tabular}

$<$ e >: valores estatisticamente significativos $(p<0,05)$ entre os grupos; TCR: treinamento contra-resistência; H: homens; M: mulheres; PLA: Placebo; CK: creatina cinase; LDH: lactato desidrogenase; M M : massa magra; M G: gordura corporal; M M SS: membro superior; M M II: membro inferior; MCT: massa corporal total; SCP: suplemento carboidrato/proteína; CR: creatina; HMB: beta-hidroxi-beta-metilbutirato; SEM : semana; NA: não analisado; sprint: corrida em velocidade máxima. Todos os estudos foram randomizados e duplo-cegos, com exceção do Hoffman et al., o qual foi simples cego

Ressalta-se que grande parte dos estudos com HMB foi publicada apenas na forma de resumos em anais de congressos, limitando a obtenção de informações mais detalhadas, principalmente, no que tange aos protocolos de treinamento contra-resistência utilizados.

Em linhas gerais, há uma carência de pesquisas aprofundadas e que utilizem indicadores metabólicos mais precisos para esclarecer 0 mecanismo fisiológico por meio do qual o HM B pode exercer seus efeitos anti-catabólicos em indivíduos destreinados.

Em relação a possíveis efeitos adversos sobre a saúde, não há registros, ao menos ao nosso conhecimento, que o HM B possa produzi-los, e o Comitê Olímpico Internacional o classifica com uma substância legal.

\section{A GRA DECIMENTOS}

Os autores agradecem a colaboração dos Professores Paulo Sergio Chagas Gomes e M ônica Lopes de Mello.

\section{COLABORADORES}

T.S. ALVARES e C.M. MEIRELLES participaram na elaboração da estratégia de busca bibliográfica e no levantamento e interpretação dos estudos e na redação do artigo.

\section{REFERÊ N CIAS}

1. Pereira RF, Lajolo FM, Hirschbruch MD. Consumo de suplementos por alunos de academias de 
ginástica em São Paulo. Rev Nutr. 2003; 16(3): 265-72.

2. American College of Sports Medicine. Position stand on progression models in resistance training for healthy adults. Med Sci Sports Exerc. 2002; 34(2):364-80.

3. Santos TM, Gomes PSC, Santos MO, Gomes UN, Ribeiro LG. Behavioral characteristics of clients enrolling in health and fitness facilities in Rio de Janeiro. Med Sci Sports Exerc. 2005; 37(5 Suppl): S359.

4. Nissen SL, Sharp RL. Effect of dietary supplements on lean mass and strength gains with resistance exercise: a meta-analysis. J Appl Physiol. 2003; 94(2):651-9.

5. Ostaszewski P, Kostiuk S, Balasinska B, Papet I, Glomot F, Nissen $S$. The effect of the leucine metabolite 3-hydroxy 3-methylbutyrate (HMB) on muscle protein synthesis and protein breakdown in chick and rat muscle [A bstract]. J Anim Sci. 1996; 74(Suppl 1):138.

6. van Koevering $M$, Nissen S. Oxidation of leucine and $\alpha$-ketoisocaproate to $\beta$-hydroxy- $\beta$ methylbutyrate in vivo. Am J Physiol. 1992; 262(1 Pt 1):E27-31.

7. Nissen S, Sharp RL, Panton L, Vukovich M, Trappe S, Fuller JC Jr. Beta-hydroxy-beta-methylbutyrate (HM B) supplementation in humans is safe and may decrease cardiovascular risk factors. J Nutr. 2000; 130(8):1937-45.

8. Nonnecke B, Franklin S, Nissen S. Leucine and its catabolites alter mitogen-stimulated DNA synthesis by bovine lymphocytes. J Nutr. 1991; 121(10): 1665-72.

9. Peterson $A L$, Qureshi $M A$, Ferket $P R$, Fuller J. In vitro exposure with b-hydroxy-b-methylbutyrate enhances macrophage growth and function. Poultry Sci. 1996; 75(1):7.

10. Ostaszewski P, Papet I, Nissen S, Glomot F, Grizard J, Amal M. Dietary supplementation of 3-hydroxy-3-methylbutirate improves catch-up growth in underfed lambs. Ann Zootech. 1994; 43:308.

11. Slater GJ, Jenkins D. Beta-hydroxy-betametylbutyrate (HMB) supplementation and the promotion of muscle grownth and strength. Sports Med. 2000; 30(2):105-16.

12. Gallagher PM, Carrithers JA, Godard MP, Schulze KE, Trappe SW. Beta-hydroxy-beta-methylbutyrate ingestion, part II: effects on hematology, hepatic and renal function. Med Sci Sports Exerc. 2000b; 32(12):2116-9.

13. Nissen S, Panton L, W ilhelm R, Fuller JC Jr. Effects of beta-hydroxy-beta-metylbutyrate (HMB) supplementation on strength and body composition of trained and untrained males undergoing intense resistance training. FASEB J. 1996a; 9(3):A287.

14. Nissen SL, Sharp R, Ray M, Rathmacher JA, Rice D, Fuller JC Jr., et al. The effect of the leucine metabolite b-hydroxy-b-methylbutyrate on muscle metabolism during resistance-exercise training. J Appl Physiol. 1996b; 81(5):2095-104.

15. Vukovich MD, Strubbs NB, Bohlken RM, Desch MF, Fuller Jr. JC, Rathmacher JA. The effect of dietary b-Hydroxy-b-M ethylbutyrate (HMB) on strength gains and body composition changes in older adults. FASEB J. 1997; 11(3):A376.

16. Vukovich MD, Stubbs NB, Bohlken RM. Body composition in 70-year-old adults responds to dietary beta-hydroxy-beta-methylbutyrate similarly to that of young adults. J Nutr. 2001; 131(7): 2049-52.

17. Panton LB, Rathmacher JA, Baier S, Nissen S. Nutritional supplementation of the leucine metabolite beta-hydroxy-beta-methylbutyrate (HMB) during resistance training. Nutrition. 2000; 16(9):734-9.

18. Knitter AE, Panton L, Rathmacher JA, Petersen A, Sharp R. Effects of $\beta$-hydroxy- $\beta$-methylbutyrate on muscle damage after a prolonged run. J Appl Physiol. 2000; 89:1340-4.

19. Nissen S, Panton L, Fuller Jr J, Rice D, Ray M, Sharp R. Effect of feeding $\beta$-hydroxy- $\beta$-methylbutyrate on body composition and strength of women. FASEB J. 1997; 10(3).

20. O'Brien C, Young AJ, Sawka MN. Bioelectrical impedance to estimate changes in hydration status. Int J Sports Med. 2002; 23(5):361-6.

21. Meirelles CM. Efeitos da nutrição sobre o metalismo protéico muscular no treinamento contra-resistência. Rev Bras Fisiol Exerc. 2004; 3(2):237-42.

22. Jówko $E$, Ostaszewski P, Jank $M$, Sacharuk J, Zieniewicz A, Wilczak J, et al. Creatine and $\beta$-hydroxy- $\beta$-methylbutyrate (HMB) additively increase lean body mass and muscle strength during a weight-training program. Nutrition. 2001; 17(7-8):558-66.

23. Hultman E, Söderlund K, Timmons JA, Cederblad G, Greenhaff PL. Muscle creatine loading in men. J Appl Physiol. 1996; 81(1):232-7.

24. O'Connor DM, Crowe MJ. Effects of beta-hydroxy-beta-methylbutyrate and creatine monohydrate supplementation on the aerobic and anaerobic capacity of highly trained athletes. J Sports Med Phys Fitness. 2003; 43(1):64-8.

25. Gallagher PM, Carrithers JA, Godard M P, Schulze KE, Trappe SW. Beta-hydroxy-beta-methylbutyrate ingestion, part I: effects on strength and fat free mass. Med Sci Sports Exerc. 2000; 32(12):2109-15. 
26. Perini TA, Oliveira GL, Ornellas JS, Oliveira FP. Cálculo do erro técnico de medição em antropometria. Rev Bras Med Esp. 2005; 11(1):81-4.

27. Talleyrand V, Zhang Z, Rathmecher J, Nissen S. Uptake and output of leucine metabolite b-hydroxy b-methyl butyrate (HMB) across the leg of pigs. FASEB J. 1993; 7(3):A71.

28.Panton L, Rathmacher J, Fuller J. Effect of $\beta$-hydroxy- $\beta$-methylbutyrate and resistance training on strength and functional ability in the elderly. Med Sci Sports Exerc. 1998; 30(5):S194.

29. Rennie MJ, Millward DJ. 3-methilhystidine excretion and the urinary 3-methylhistidine/ creatinine ratio are poor indicators of skeletal muscle protein breakdown. Clin Sci. 1983; 65(3): 217-25.

30. Sorichter S, Puschendorf B, M air J. Skeletal muscle injury induced by eccentric muscle action: muscle proteins as markers of muscle fiber injury. Exerc Immunol Rev. 1999; 5:5-21.

31. Ransone J, Neighbors K, LeFavi R, Chromiak J. The effect of $b$-Hydroxy-b-M ethylbutyrate on muscular strength and body composition in collegiate football players. J Strength Cond Res. 2003; 17(1): 34-9.

32. Kreider RB, Ferreira M, W ilson M, Grindstaff P, Plisk $S$, Reinhard J, et al. Effects of calcium $\beta$-HMB supplementation with or without creatine during training on body composition alterations. FASEB J. 1997; 11(3):A374.

33. Kreider RB, Ferreira $M$, Greenwood $M$, Wilson $M$, Grindstaff P, Plisk S, et al. Effects of calcium $\beta$-HMB supplementation during training on markers of catabolism, body composition, strength and sprint performance. JEPonline. 2000; 3(4):48-59.

34. Slater GJ, Jenkins $D$, Logan $P$, Lee $H$, Vukovich $M$, Rathmacher JA, et al. Beta-hydroxy-betamethylbutyrate (HMB) supplementation does not affect changes in strength or body composition during resistance training in trained men. Int J Sport Nutr Exerc Metab. 2001; 11(3):384-96.

35. Thomson JS. $\beta$-Hydroxy- $\beta$-M ethylbutyrate (HMB) supplementation of resistance trained men. Asia Pac J Clin Nutr. 2004; 28(13 Suppl):S59.

36. Phillips SM, Parise G, Roy BD, Tipton KD, Tarnopolsky MA. Resistance-training induced adaptations in skeletal muscle protein turnover in the fed state. Can J Physiol Pharmacol. 2002; 80(11):1045-53.

37. Kreider RB, Ferreira M, Wilson M, Almada AL. Effects of calcium beta-hydroxy-beta-metylbutyrate (HM B) supplementation during resistance-training on makers of catabolism, body composition and strength. Int J Sports Med. 1999b; 20(8)503-9.

38. Hoffman JR, Cooper J, Wendell M, Im J, Kang J. Effects of beta-hydroxy beta-methylbutyrate on power performance and indices of muscle damage and stress during high-intensity training. J Strength Cond Res. 2004; 18(4):747-52.

Recebido em: 2/8/2006

Versão final reapresentada em: 24/11/2006 Aprovado em: 27/7/2007 
